\title{
THURSTON'S METRIC ON TEICHMÜLLER SPACE AND THE TRANSLATION DISTANCES OF MAPPING CLASSES
}

\author{
Athanase Papadopoulos and Weixu Su \\ Université de Strasbourg and CNRS, Institut de Recherche Mathématique Avancée \\ 7 rue René Descartes, 67084 Strasbourg Cedex, France; athanase.papadopoulos@math.unistra.fr \\ Fudan University, School of Mathematics \\ 200433, Shanghai, P. R. China; suwx@fudan.edu.cn
}

\begin{abstract}
We show that the Teichmüller space of a surface without boundary and with punctures, equipped with the Thurston metric, is the limit in an appropriate sense of Teichmüller spaces of surfaces with boundary, equipped with their arc metrics, when the boundary lengths tend to zero. We use this to obtain a result on the translation distances of mapping classes for their actions on Teichmüller spaces equipped with the Thurston metric.
\end{abstract}

\section{Introduction}

In this paper, we show that the arc metrics on the Teichmüller space of surfaces with boundary limit to the Thurston metric on the Teichmüller space of a surface without boundary, by making the boundary lengths tend to zero. We use this to prove a result on the translation distances for mapping classes.

We introduce some notation before stating precisely the results. In all this paper, $S=S_{g, p, n}$ is a connected orientable surface of finite type, of genus $g$ with $p$ punctures and $n$ boundary components. We assume that $S$ has negative Euler characteristic, i.e., $\chi(S)=2-2 g-p-n<0$. When $n>0$, we denote by $\partial S$ the boundary of $S$. A hyperbolic structure on $S$ is a complete metric of constant curvature -1 such that

(i) each puncture has a neighborhood isometric to a cusp, i.e., to the quotient

$$
\left\{z=x+i y \in \mathbf{H}^{2} \mid y>a\right\} /\langle z \mapsto z+1\rangle,
$$

for some $a>0$,

(ii) each boundary component is a simple closed geodesic.

We denote by $\mathcal{T}(S)$ the Teichmüller space of $S$, that is, the set of homotopy classes of hyperbolic structures on this surface. We say that a simple closed curve on $S$ is essential if it is neither homotopic to a point nor to a puncture (but it can be homotopic to a boundary component).

Let $\mathcal{S}$ denote the set of homotopy classes of essential simple closed curves on $S$. An $\operatorname{arc}$ on $S$ is the homeomorphic image of a closed interval which is properly embedded in $S$, that is, the interior of the arc is in the interior of $S$ and the endpoints of the arc are on the boundary of $S$. All homotopies of arcs that we consider are relative to $\partial S$, that is, they keep the endpoints of the arc on the boundary $\partial S$ (but they do not necessarily fix pointwise the points of $\partial S$ ). An arc is said to be essential if it is not homotopic to an arc whose image is contained in $\partial S$.

doi:10.5186/aasfm.2016.4158

2010 Mathematics Subject Classification: Primary 32G15, 30F60.

Key words: Teichmüller space, hyperbolic geometry, quasiconformal mapping, Thurston metric, arc metric, translation distance, mapping class group, pseudo-Anosov, reducible. 
Let $\mathcal{A}$ denote the set of homotopy classes of essential arcs on $S$. Assume that $S$ is equipped with a hyperbolic structure $X$. For any $\gamma \in \mathcal{A} \cup \mathcal{S}$, there is a unique geodesic $\gamma^{X}$ in its homotopy class. It is orthogonal to $\partial X$ at each endpoint, in the case where $\gamma$ is an equivalence class of arcs. We denote by $\ell_{\gamma}(X)$ the length of $\gamma^{X}$, and we call it the geodesic length of $\gamma$ on $X$. This geodesic length only depends on the equivalence class of $X$ in $\mathcal{T}(S)$. Therefore it is a function defined on $\mathcal{T}(S)$.

The arc metric on $\mathcal{T}(S)$ is an asymmetric metric defined by

$$
d(X, Y)=\sup _{\gamma \in \mathcal{A} \cup \mathcal{S}} \log \frac{\ell_{\gamma}(Y)}{\ell_{\gamma}(X)} .
$$

This metric was introduced in [8]. It is an analogue for surfaces with boundary of the Thurston asymmetric metric [16]. The arc metric is also studied in the papers [2] [9] [13] [14].

By gluing a hyperbolic surface with its mirror image along the boundary components, we obtain a natural isometric embedding of the Teichmüller space $\mathcal{T}(S)$ into $\mathcal{T}(\widetilde{S})$, where $\widetilde{S}$ denotes the double of $S$. We endow $\mathcal{T}(\widetilde{S})$ with the Thurston metric. The authors do not know whether such an embedding is totally geodesic (although this is unlikely). In a recent paper [2], the authors described how such an embedding extends continuously to the Thurston compactification. Inspired by the work of Walsh [17], the authors proved in [2] that the horofunction boundary of $(\mathcal{T}(S), d)$ is homeomorphic to the Thurston boundary. It is conjectured in [2] that, with the usual exceptional cases, the isometry group of $(\mathcal{T}(S), d)$ is the extended mapping class group.

1.1. Convergence of the arc metric. The first aim of this paper is to study the subsets of $\mathcal{T}(S)$ corresponding to hyperbolic surfaces whose boundary components have fixed lengths. These subsets are intersections of level sets of the length functions of boundary components.

Let $\mathcal{B}=\left\{\beta_{1}, \cdots, \beta_{n}\right\}$ be the set of boundary components of $S$. Given any $L=\left(L_{1}, \cdots, L_{n}\right), L_{i}>0$, we set

$$
\mathcal{T}_{L}(S)=\left\{X \in \mathcal{T}(S) \mid \ell_{\beta_{i}}(X)=L_{i}, 1 \leq i \leq n\right\}
$$

For a fixed vector $L=\left(L_{1}, \cdots, L_{n}\right)$, there is a metric on $\mathcal{T}_{L}(S)$, defined using the same formula (1). This is the arc metric on $\mathcal{T}_{L}(S)$. It is also the metric induced by the arc metric of $\mathcal{T}(S)$ on $\mathcal{T}_{L}(S)$ considered as a subset of $\mathcal{T}(S)$.

When $L=0$, we define $\mathcal{T}_{0}(S)$ be the Teichmüller space of hyperbolic structures on $S$ such that each boundary component is replaced by a puncture. We prove that as $L \rightarrow 0$, the restriction of the arc metric on $\mathcal{T}_{L}(S)$ limits to the Thurston metric on $\mathcal{T}_{0}(S)$. This limiting behaviour is described using Fenchel-Nielsen coordinates. It is also expressed in Proposition 2.3 below where we prove that there is a certain map $\Psi_{L}: \mathcal{T}_{L}(S) \rightarrow \mathcal{T}_{0}(S)$ which is a $(1, o(1))$-quasi-isometry.

1.2. Application to translation distances in the mapping class group. In the second part of this paper we use the results of the first part to study the translation distances of mapping class group elements on Teichmüller space equipped with the Thurston metric. This is based on Proposition 2.3. Let us be more precise.

In this part, the surface is without boundary, that is, $S$ is an oriented surface obtained from a closed surface by removing finitely many points (possibly none). We denote its mapping class group by $\operatorname{Mod}(S)$. For an element $f \in \operatorname{Mod}(S)$, its 
translation distance with respect to the Thurston metric $d_{\mathrm{Th}}$ on $\mathcal{T}(S)$ is defined by

$$
\sigma(f)=\inf _{X \in \mathcal{T}(S)} d_{\mathrm{Th}}(X, f(X)) .
$$

The maximal dilatation of $f$ is the largest of the dilatation constants of its pseudoAnosov components, with respect to the Thurston decomposition of this mapping class. See [7] and [15] for the decomposition of a mapping class.

We prove the following:

Theorem 1.1. Let $f \in \operatorname{Mod}(S)$ and $\lambda(f)$ be the maximal dilatation of $f$. Then there exists an integer $n$ such that

$$
\sigma\left(f^{n}\right)=\log \lambda\left(f^{n}\right)
$$

Note that we always have $\log \lambda\left(f^{n}\right)=n \log \lambda(f)$. We recall that Bers proved in [4] that

$$
\log \lambda(f)=\inf _{X \in \mathcal{T}(S)} d_{\text {Teich }}(X, f(X)),
$$

where $d_{\text {Teich }}$ denotes the Teichmüller metric.

Recall that from Thurston's classification, the mapping class $f$ is either periodic, reducible or pseudo-Anosov. When $f$ is periodic, it has a fixed point in $\mathcal{T}(S)$, thus $\sigma(f)=0$ and (2) is trivial. Theorem 1.1 was proved in [10] when $f$ is pseudo-Anosov. In the present paper, we prove Theorem 1.1 by showing that when $f$ is reducible, one can decrease the distance $d_{\mathrm{Th}}(X, f(X))$ (not necessary strictly) by taking a sequence of points $X \in \mathcal{T}(S)$ such that the lengths of the reducible curves of $f$ decrease to zero.

Remark 1.2. In the paper [10], the authors announced a proof that $\sigma(f)=$ $\log \lambda(f)$ for any pseudo-Anosov mapping class $f$. Unfortunately, there is a gap in the argument. In fact, the (weaker) result which is proved there is that this holds up to taking a power of $f$. This is also a particular case of Theorem 1.1 of the present paper.

The difficulty to understand the translation distance $\sigma(f)$ lies in the fact that the Thurston metric is not uniquely geodesic. However, there is another notion of translation distance obtained by setting:

$$
\tau(f)=\lim _{n \rightarrow \infty} \frac{1}{n} d_{\mathrm{Th}}\left(X_{0}, f^{n}\left(X_{0}\right)\right),
$$

where $X_{0} \in \mathcal{T}(S)$ is fixed. It is not hard to check that the definition of $\tau(f)$ is independent on the choice of $X_{0}$. As a corollary of Theorem 1.1, we have

Corollary 1.3. Let $f \in \operatorname{Mod}(S)$ and $\lambda(f)$ be the maximal dilatation of $f$. Then $\tau(f)=\log \lambda(f)$.

Acknowledgements. The authors are partially supported by the French ANR grant FINSLER (Géométrie de Finsler et applications, ANR-12-BS01-0009). W. Su is partially supported by NSFC No: 11201078. The authors wish to thank the referee for his/her remarks and corrections.

\section{Convergence of the arc metric under pinching}

In this section, $S=S_{g, p, n}$ is a connected orientable surface of genus $g$ with $p$ punctures and $n$ boundary components, and $\mathcal{B}=\left\{\beta_{1}, \cdots, \beta_{n}\right\}$ is the set of boundary components of $S$. Let $\mathcal{C}=\left\{\gamma_{1}, \cdots, \gamma_{3 g-3+p}\right\}$ be a maximal collection of distinct homotopy classes of disjoint essential simple closed curves in the interior of $S$. The 
union $\mathcal{C} \cup \mathcal{B}$ is a pants decomposition of $S$. We denote the corresponding FenchelNielsen (length-twist) coordinates on $\mathcal{T}(S)$ by

$$
\left(\left(\ell_{1}, \tau_{1}\right), \cdots,\left(\ell_{3 g-3+p}, \tau_{3 g-3+p}\right)\right) \times\left(\ell_{3 g-3+p+1}, \cdots, \ell_{3 g-3+p+n}\right) .
$$

Let us fix $L=\left(\ell_{3 g-3+p+1}, \cdots, \ell_{3 g-3+p+n}\right)$. We define a map

$$
\begin{aligned}
\Psi_{L}: \mathcal{T}_{L}(S) & \rightarrow T_{0}(S), \\
\left(\left(\ell_{1}, \tau_{1}\right), \cdots,\left(\ell_{3 g-3+p}, \tau_{3 g-3+p}\right)\right) \times L & \mapsto\left(\left(\ell_{1}, \tau_{1}\right), \cdots,\left(\ell_{3 g-3+p}, \tau_{3 g-3+p}\right)\right)
\end{aligned}
$$

It is clear that $\Psi_{L}$ is a homeomorphism.

Definition 2.1. We say that a family of hyperbolic surfaces $X_{L} \in \mathcal{T}_{L}(S)$ converges to a point $X_{0} \in \mathcal{T}_{0}(S)$, and we denoted this relation by $X_{L} \rightarrow X_{0}$, if $\Psi_{L}\left(X_{L}\right) \rightarrow X_{0}$ as $L \rightarrow 0$.

The definition is independent of the choice of the pants decomposition. The reason is that, for any given simple closed geodesic $\gamma$ in the interior of $X_{L}$, we can explicitly compute the hyperbolic length and twist angle of $\gamma$ by using the FenchelNielsen coordinates $\left(\left(\ell_{1}, \tau_{1}\right), \cdots,\left(\ell_{3 g-3+p}, \tau_{3 g-3+p}\right)\right) \times L$ of $X_{L}$. (For the explicit formulae, see [6].) When $X_{L} \rightarrow X_{0}$ as defined here, then, as $L \rightarrow 0$, the hyperbolic length and twist angle of $\gamma$ converge to those of $\gamma$ on $X_{0}$. As a result, given any two different pants decompositions, there is a continuous transformation between their associated Fenchel-Nielsen coordinates. In fact, this convergence is similar to that in augmented Teichmüller space. If we take the double of each Riemann surface, $X_{L}$, then the sequence of doubles converges to a noded Riemann surface, which is the double of $X_{0}$. It is well known that the convergence of a sequence in the augmented Teichmüller space can be described using Fenchel-Nielsen coordinates, and that this convergence is independent of the choice of Fenchel-Nielsen coordinates (cf. [1] or [19]).

We shall use the following convergence criterion proved by Mondello; cf. [12, Theorem 7.1] for a more general statement. In this statement, $g_{L}$ denotes the hyperbolic metric of $X_{L} \in \mathcal{T}_{L}(S)$.

Lemma 2.2. $X_{L} \rightarrow X_{0}$ if and only if there exist homeomorphisms $f_{L}: X_{0} \rightarrow X_{L}$ such that $\left(f_{L}\right)^{*}\left(g_{L}\right) \rightarrow g_{0}$ uniformly on the compact subsets of $X_{0}$.

Here, $\left(f_{L}\right)^{*}\left(g_{L}\right)$ denotes the pull-back of the metric $g_{L}$ on $X_{0}$ by the map $f_{L}$ and we say that $\left(f_{L}\right)^{*}\left(g_{L}\right) \rightarrow g_{0}$ uniformly on the compact subsets of $X_{0}$ if for every compact subset $K \subset X_{0}$, the restriction of the metric $\left(f_{L}\right)^{*}\left(g_{L}\right)$ on $K$ (in the sense of the metric tensors or the quadratic forms on the tangent spaces) converges uniformly to the restriction of $g_{0}$ on $K$, as $L \rightarrow 0$. Note that this is equivalent to say that, with restriction on $K,\left(f_{L}\right)^{*}\left(g_{L}\right)$ and $g_{0}$ are bi-Lipschitz equivalent with a Lipschitz constant tending to 1 as $L \rightarrow 0$.

2.1. The Thurston metric as a limit of the arc metric. We are only interested in the situation where $L$ is sufficiently small. We write in this case $L \ll 1$. If a quantity $A \rightarrow \infty$ uniformly as $L \rightarrow 0$, we write $A \gg 1$. A quantity $B=B(L)$ satisfying $B \rightarrow 0$ as $L \rightarrow 0$ is denoted by $o(1)$.

Recall that the Thurston metric on $\mathcal{T}_{0}(S)$ is defined by

$$
d_{\mathrm{Th}}(X, Y)=\sup _{\gamma \in \mathcal{S}} \log \frac{\ell_{\gamma}(Y)}{\ell_{\gamma}(X)} .
$$

Using the notation established at the beginning of this section, we prove the following: 
Proposition 2.3. For each $L \ll 1$, the map $\Psi_{L}: \mathcal{T}_{L}(S) \rightarrow \mathcal{T}_{0}(S)$ is a $(1, o(1))$ quasi-isometry.

More precisely, we have, for any $L$ and for any $X$ and $Y$ in $\mathcal{T}_{L}(S)$,

$$
d(X, Y)-o(1) \leq d_{\mathrm{Th}}\left(\Psi_{L}(X), \Psi_{L}(Y)\right) \leq d(X, Y)+o(1) .
$$

Proof. Let $X_{0} \in \mathcal{T}_{0}(S)$. We denote by $X_{L}$ the set $\Psi_{L}^{-1}\left(X_{0}\right)$. It is obvious that $X_{L} \rightarrow X_{0}$ as $L \rightarrow 0$ in the sense of Definition 2.1.

First, we estimate the lengths of simple closed curves $\ell_{\gamma}\left(X_{L}\right)$ in terms of $\ell_{\gamma}\left(X_{0}\right)$. For any boundary component $\beta$ of $S$, we denote its unique geodesic representative in $X_{L}$ by $\beta_{L}$. By a well-known result [6], there is a collar neighborhood of $\beta_{L}$ of width $w\left(\beta_{L}\right)$ such that

$$
\sinh \frac{\ell_{\beta}\left(X_{L}\right)}{2} \sinh w\left(\beta_{L}\right)=1 .
$$

Note that $w\left(\beta_{L}\right) \rightarrow \infty$ as $L \rightarrow 0$. It is also well known that a simple closed geodesic on $X_{L}$ that does not intersect $\beta_{L}$ is disjoint from such a collar [18]. In particular, any simple closed geodesic in the interior of $X_{L}$ is disjoint from the collar.

Similarly, we choose a standard cusp neighborhood of each puncture of $X_{0}$, that is, a neighborhood isometric to

$$
\left\{\Im z>\frac{1}{2}\right\} /\langle z \mapsto z+1\rangle .
$$

Any simple closed geodesic on $X_{0}$ is disjoint from the standard cusp neighborhood.

Denote the union of the standard cusp neighborhoods on $X_{0}$ by $C$. Using Lemma 2.2 , it is not hard to see that there exist homeomorphisms $f_{L}: X_{0} \rightarrow X_{L}$ such that (as $L \ll 1)$

(i) $\left(f_{L}\right)^{*}\left(g_{L}\right) \rightarrow g_{0}$ uniformly on $X_{0} \backslash C$;

(ii) any closed geodesic in the interior of $X_{L}$ is contained in $X_{L} \backslash f_{L}^{-1}(C)$. As a result, we have

$$
\ell_{\gamma}\left(X_{L}\right)=(1+o(1)) \ell_{\gamma}\left(X_{0}\right), \forall \gamma \in \mathcal{S} \backslash \mathcal{B} .
$$

The next step is to estimate the length of an essential geodesic arc on $X_{L}$. Consider an arbitrary $\alpha \in \mathcal{A}$. Suppose that $\alpha$ joins two boundary components $\beta_{1}, \beta_{2}$ of $X_{L}$ (we may have $\beta_{1}=\beta_{2}$ ). A tubular neighborhood of $\alpha \cup \beta_{1} \cup \beta_{2}$ is a topological pair of pants, denoted by $\mathcal{P}$.

We first assume that $\beta_{1} \neq \beta_{2}$. Then the boundary of $\mathcal{P}$ has three connected components, two of them being (homotopic to) $\beta_{1}$ and $\beta_{2}$. We denote by $\gamma$ the (homotopy class of the) third one.

To simplify our notation, we set $\ell_{\alpha}=\ell_{\alpha}\left(X_{L}\right)$, etc. We use the following hyperbolic geometry formula [2]:

$$
\cosh \left(\ell_{\alpha}\right)=\frac{\cosh \left(\frac{1}{2} \ell_{\gamma}\right)+\cosh \left(\frac{1}{2} \ell_{\beta_{1}}\right) \cosh \left(\frac{1}{2} \ell_{\beta_{2}}\right)}{\sinh \left(\frac{1}{2} \ell_{\beta_{1}}\right) \sinh \left(\frac{1}{2} \ell_{\beta_{2}}\right)} .
$$

By assumption, $\ell_{\beta_{1}}, \ell_{\beta_{2}} \ll 1$. By a direct calculation, we get

$$
\begin{aligned}
\ell_{\alpha}= & \log \left(\cosh \left(\ell_{\alpha}\right)+\sqrt{\cosh ^{2}\left(\ell_{\alpha}\right)-1}\right) \\
= & \log \left(\cosh \left(\frac{1}{2} \ell_{\gamma}\right)+\cosh \left(\frac{1}{2} \ell_{\beta_{1}}\right) \cosh \left(\frac{1}{2} \ell_{\beta_{2}}\right)\right) \\
& -\log \left(\sinh \left(\frac{1}{2} \ell_{\beta_{1}}\right) \sinh \left(\frac{1}{2} \ell_{\beta_{2}}\right)\right)+o(1) \\
= & \ell_{\gamma}+\left|\log \left(\sinh \left(\frac{1}{2} \ell_{\beta_{1}}\right) \sinh \left(\frac{1}{2} \ell_{\beta_{2}}\right)\right)\right|-\log 2+o(1) .
\end{aligned}
$$




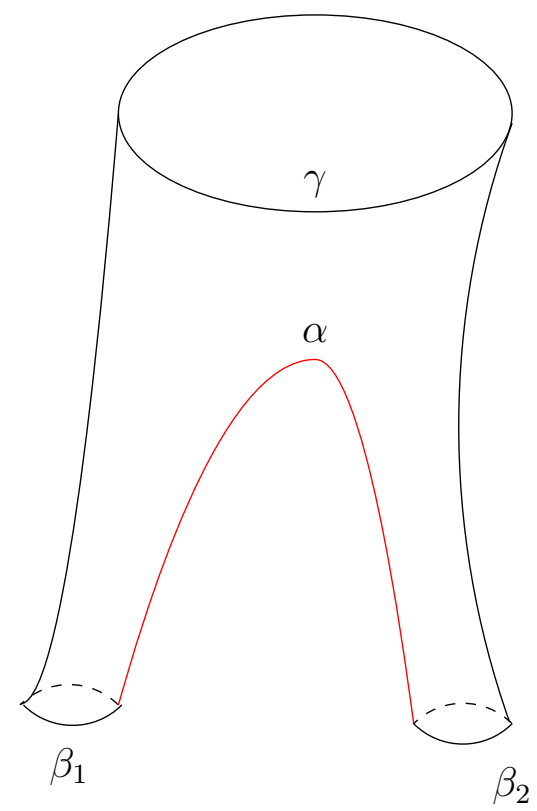

Figure 1. The regular neighborhood is homotopic to a pair of pants.

The case where $\beta_{1}=\beta_{2}$ can be dealt with in the same way, by using the following formula [2]:

$$
\begin{aligned}
\cosh ^{2}\left(\frac{1}{2} \ell_{\alpha}\right)= & \frac{-1+\cosh ^{2}\left(\frac{1}{2} \ell_{\beta}\right)+\cosh ^{2}\left(\frac{1}{2} \ell_{\gamma_{1}}\right)+\cosh ^{2}\left(\frac{1}{2} \ell_{\gamma_{2}}\right)}{\sinh ^{2}\left(\frac{1}{2} \ell_{\beta}\right)}+ \\
& +\frac{2 \cosh \left(\frac{1}{2} \ell_{\beta}\right) \cosh \left(\frac{1}{2} \ell_{\gamma_{1}}\right) \cosh \left(\frac{1}{2} \ell_{\gamma_{2}}\right)}{\sinh ^{2}\left(\frac{1}{2} \ell_{\beta}\right)}
\end{aligned}
$$

Here the boundary of the pair of pants $\mathcal{P}$ has three connected components, one of them being $\beta_{1}=\beta_{2}$. We denote by $\gamma_{1}, \gamma_{2}$ the two others.

Let $Y_{0} \in \mathcal{T}_{0}(S), Y_{0} \neq X_{0}$. Denote by $Y_{L}=\Psi_{L}^{-1}\left(Y_{0}\right)$. Applying (3), we get

$$
\frac{\ell_{\gamma}\left(Y_{L}\right)}{\ell_{\gamma}\left(X_{L}\right)}=(1+o(1)) \frac{\ell_{\gamma}\left(Y_{0}\right)}{\ell_{\gamma}\left(X_{0}\right)}, \forall \gamma \in \mathcal{S}
$$

Using the fact that

$$
\frac{A_{1}+A_{2}}{B_{1}+B_{2}} \leq \max \left\{\frac{A_{1}}{B_{1}}, \frac{A_{2}}{B_{2}}\right\}, \quad A_{1}, A_{2}, B_{1}, B_{2}>0
$$

we derive from (4) that for any $\alpha \in \mathcal{A}$, there is some $\gamma \in \mathcal{S}$ such that

$$
\frac{\ell_{\alpha}\left(Y_{L}\right)}{\ell_{\alpha}\left(X_{L}\right)} \leq \max \left\{\frac{\ell_{\gamma}\left(Y_{L}\right)}{\ell_{\gamma}\left(X_{L}\right)}, 1+o(1)\right\} \leq(1+o(1)) \max \left\{\frac{\ell_{\gamma}\left(Y_{0}\right)}{\ell_{\gamma}\left(X_{0}\right)}, 1\right\} .
$$

In conclusion, we have

$$
\left|d\left(X_{L}, Y_{L}\right)-d_{\mathrm{Th}}\left(X_{0}, Y_{0}\right)\right|=o(1) .
$$

This proves the proposition.

Remark 2.4. Instead of studying only the convergence of hyperbolic structures $X_{L}$ to a hyperbolic structure $X_{0}$ where all boundary components become cusps (length zero), one may study convergence to hyperbolic structures where some of the boundary components are cusps. The same arguments we used, but with heavier notation, show that we may view the spaces $\mathcal{T}_{L}$ associated with fixed vectors $L$ with 
some zero coordinates as sitting on the boundary of the Teichmüller space of the surface $S=S_{g, p, n}$ with $p$ punctures and $n$ boundary components. This boudary has an obvious stratified structure. The convergence to the boundary is in the sense of the arc metrics (each stratum is endowed with its own arc metric). The arc metrics, as we prove in the present paper, converge to the Thurston metric as one approaches surfaces $X_{0}$ where all the boundary components become cusps.

\section{Translation distances of mapping classes}

We now consider the Teichmüller space $\mathcal{T}(S)$ as a space of equivalence classes of marked hyperbolic structures $(X, g)$, where $X$ is a hyperbolic surface and $g: S \rightarrow$ $X$ an orientation-preserving homeomorphism (rather than a space of equivalence classes of metrics on a fixed surface). Two marked hyperbolic structures $\left(X_{1}, g_{1}\right)$ and $\left(X_{2}, g_{2}\right)$ are equivalent if and only if there is a conformal mapping $h: X_{1} \rightarrow X_{2}$ in the homotopy class of $g_{2} \circ g_{1}^{-1}$. With this definition, a mapping class $f$ acts on $\mathcal{T}(S)$ by changing the markings

$$
f:(X, g) \mapsto(X, g \circ f) .
$$

In this section, the surface $S$ is without boundary. Its Teichmüller space is endowed with the Thurston metric $d_{\mathrm{Th}}$. We recall that the translation distance of an element $f \in \operatorname{Mod}(S)$ is defined by

$$
\sigma(f)=\inf _{X \in \mathcal{T}(S)} d_{\mathrm{Th}}(X, f(X)) .
$$

3.1. Reducible maps. We first show that the question of understanding $\sigma(f)$ for any $f \in \operatorname{Mod}(S)$ can be reduced to that of a pseudo-Anosov mapping class $f$. We shall pass to surfaces with boundary, those obtained by cutting $S$ along a complete reducing system for the mapping class $f$. We shall equip the Teichmüller spaces of these surfaces with boundary with the arc metric and use the result of the preceding section.

Assume that $f \in \operatorname{Mod}(S)$ is reducible. Let $\mathcal{C}=\left\{c_{1}, \cdots, c_{r}\right\} \subset \mathcal{S}$ be the maximal set of disjoint simple closed curves such that $f(\mathcal{C})=\mathcal{C}$. We denote the connected components of $S \backslash \mathcal{C}$ by $R^{1}, \cdots, R^{s}$. Each $R^{j}, 1 \leq j \leq s$, is a surface of negative Euler characteristic with finitely many boundary components. We choose an arbitrary hyperbolic structure on $S$ such that the length of the system $\mathcal{C}=\left\{c_{1}, \cdots, c_{r}\right\}$ is $L=\left(L_{1}, \cdots, L_{r}\right)$ and we assume that $L_{1}=\cdots=L_{r}$, as a matter of convenience. We shall no more use the notation $L_{j}$ in this setting. Note that the existence of such a hyperbolic structure $X_{L}$ is obvious, and that such a structure is not unique, since one can perform twists around the curves in $\mathcal{C}$. We let $\left\{X_{L}\right\}$ be the family of hyperbolic structures on $S$ corresponding to a given $L$.

In the following, convergence $X_{L} \rightarrow X_{0}$ is in the sense of Definition 2.1. The restriction of each $X_{L}$ to each subsurface $R^{j}, 1 \leq j \leq s$, induces a hyperbolic structure on $R^{j}$, which we denote by $X_{L}^{j}$. The following lemma reduces the study of $\sigma(f)$ to the consideration of a "smaller" Teichmüller space.

Lemma 3.1. Assume that $X_{L} \rightarrow X_{0}$. As $L \rightarrow 0$, we have $X_{L}^{j} \rightarrow X_{0}^{j}, 1 \leq j \leq s$ and

$$
d_{\mathrm{Th}}\left(X_{L}, f\left(X_{L}\right)\right) \rightarrow \max _{j}\left\{d_{\mathrm{Th}}\left(X_{0}^{j}, f\left(X_{0}^{j}\right)\right)\right\}
$$

Proof. It is clear that $X_{L}^{j} \rightarrow X_{0}^{j}, 1 \leq j \leq s$. Moreover, the proof of Lemma 2.3 implies that

$$
\liminf _{L \rightarrow 0} d_{\mathrm{Th}}\left(X_{L}, f\left(X_{L}\right)\right) \geq \max _{j}\left\{d_{\mathrm{Th}}\left(X_{0}^{j}, f\left(X_{0}^{j}\right)\right)\right\}
$$


It remains to show that

$$
\limsup _{L \rightarrow 0} d_{\operatorname{Th}}\left(X_{L}, f\left(X_{L}\right)\right) \leq \max _{j}\left\{d_{\mathrm{Th}}\left(X_{0}^{j}, f\left(X_{0}^{j}\right)\right)\right\} .
$$

The proof given below is similar to our argument in the proof of Proposition 2.3.

Let $\gamma \in \mathcal{S}$. If $\gamma$ is disjoint from $\mathcal{C}$, then $\gamma$ is contained in some subsurface $R^{j}$. By Lemma $2.2, \ell_{\gamma}\left(X_{L}\right)=(1+o(1)) \ell_{\gamma}\left(X_{0}^{j}\right)$. This implies that

$$
\sup _{\gamma \in \mathcal{S}, i(\gamma, \mathcal{C})=0} \log \frac{\ell_{\gamma}\left(f\left(X_{L}\right)\right)}{\ell_{\gamma}\left(X_{L}\right)} \leq \max _{j}\left\{d_{\mathrm{Th}}\left(X_{0}^{j}, f\left(X_{0}^{j}\right)\right)\right\} .
$$

Now we assume that $\gamma \in \mathcal{S}$ and $i(\gamma, \mathcal{C}) \neq 0$. On the hyperbolic surface $X_{L}, \mathcal{C}$ is isotopic to a geodesic submanifold (the geodesic representative of $\left\{c_{1}, \cdots, c_{r}\right\}$ ), denoted by $\widehat{\mathcal{C}}$. Up to isotopy, we may assume that $f(\widehat{\mathcal{C}})=\widehat{\mathcal{C}}$. The geodesic representative of $\gamma$ on $X_{L}$ is decomposed by $\widehat{\mathcal{C}}$ into finite many geodesic segments, denoted by $\gamma_{1}, \cdots, \gamma_{k}$. The mapping class $f$ acts on $X_{L}$ by pull-back (which, restricted to $\widehat{\mathcal{C}}$, is an isometry). If we look at the geodesic representative of $\gamma$ on $f\left(X_{L}\right)$, it is also decomposed by $\widehat{\mathcal{C}}$ into geodesic segments; each of them will be denoted by $\gamma_{j}^{\prime}$, corresponding to $\gamma_{j}$. We denote the length of each $\gamma_{j}$ and $\gamma_{j}^{\prime}$ by $L_{j}$ and $L_{j}^{\prime}$, respectively.

We assume that $\gamma_{j}$ is contained in some subsurface $X_{L}^{s(j)}$, which is identified to a point in the Teichmüller space $\mathcal{T}\left(R_{s(j)}\right)$ of the subsurface $R_{s(j)}$. Since the mapping class $f$ acts on $\mathcal{T}\left(R_{s(j)}\right)$, we denote the image of $X_{L}^{s(j)}$ by $f\left(X_{L}^{s(j)}\right)$. We denote by $\ell_{j}=\ell\left(\gamma_{j}, X_{L}^{s(j)}\right)$, the length of the geodesic representative of the arc $\gamma_{j}$ on $X_{L}^{s(j)}$, and $\ell_{j}^{\prime}=\ell\left(\gamma_{j}, f\left(X_{L}^{s(j)}\right)\right)$. Note that the distinction between $\ell_{j}$ and $L_{j}$ is that, to define $\ell_{j}$, the endpoints of the arc $\gamma_{j}$ are allowed to vary on the boundary curves of the subsurface and $\ell_{j}$ is taken as the minimum, whereas for $L_{j}$ the endpoints are not allowed to vary. We claim that:

Lemma 3.2. As $L$ is sufficiently small,

$$
\frac{L_{j}^{\prime}}{L_{j}} \leq(1+o(1)) \max \left\{1, \frac{\ell_{j}^{\prime}}{\ell_{j}}\right\}
$$

The proof of Lemma 3.2 is postponed to $§ 3.3$. Assuming this lemma, we continue the proof of Lemma 3.1. Using (6), we have

$$
\frac{\ell_{\gamma}\left(f\left(X_{L}\right)\right)}{\ell_{\gamma}\left(X_{L}\right)}=\frac{\sum L_{j}^{\prime}}{\sum L_{j}} \leq(1+o(1)) \max \left\{1, \frac{\ell\left(\gamma_{j}, f\left(X_{L}^{s(j)}\right)\right)}{\ell\left(\gamma_{j}, X_{L}^{s(j)}\right)}\right\} .
$$

As a result, we have

$$
\sup _{\gamma \in \mathcal{S}, i(\gamma, \mathcal{C}) \neq 0} \log \frac{\ell_{\gamma}\left(f\left(X_{L}\right)\right)}{\ell_{\gamma}\left(X_{L}\right)} \leq \max _{1 \leq j \leq s}\left\{d\left(X_{L}^{j}, f\left(X_{L}^{j}\right)\right)\right\}+o(1) .
$$

By Proposition 2.3, the right-hand side converges to

$$
\max \left\{d_{\mathrm{Th}}\left(X_{0}^{j}, f\left(X_{0}^{j}\right)\right\} .\right.
$$

The lemma follows from this result and (5).

There is a converse construction. We can first pick a point $\left(X_{0}^{1}, \cdots, X_{0}^{s}\right)$ in the product of Teichmüller spaces $\mathcal{T}_{0}(S \backslash \mathcal{C}):=\prod_{j} \mathcal{T}_{0}\left(R^{j}\right)$, and construct a family of hyperbolic structures $X_{L}$ in $\mathcal{T}(S)$ such that $X_{L}^{j} \rightarrow X_{0}^{j}$. As a result, we obtain:

Corollary 3.3. If $f \in \operatorname{Mod}(S)$ is reduced by $\mathcal{C}=\left\{c_{1}, \cdots, c_{r}\right\} \subset \mathcal{S}$, then $\sigma(f)$ is at most the translation distance of the action by $f$ restricted on $\mathcal{T}_{0}(S \backslash \mathcal{C})$. 
Let $R^{0}$ be a connected component of $S \backslash \mathcal{C}$. There is a smallest integer $k$ such that $f^{k}\left(R^{0}\right)=R^{0}$. We say that the union $\mathcal{R}=R_{0} \cup f\left(R_{0}\right) \cup \cdots \cup f^{k-1}\left(R_{0}\right)$ is a reducible component of $f$. We set

$$
\mathcal{T}(\mathcal{R})=\prod_{j=0}^{k-1} \mathcal{T}_{0}\left(f^{j}\left(R_{0}\right)\right)
$$

and we endow this space with the supremum Thurston metric, which we still denote by $d_{\mathrm{Th}}$. We set

$$
\sigma_{\mathcal{R}}(f)=\inf _{X \in \mathcal{T}(\mathcal{R})} d_{\mathrm{Th}}(X, f(X)) .
$$

By Corollary 3.3, we have

$$
\sigma(f) \leq \max _{\mathcal{R}} \sigma_{\mathcal{R}}(f)
$$

Note that the action of $f$ on $\mathcal{R}$ is either periodic or pseudo-Anosov. When $\mathcal{R}$ is a periodic component of $f, \sigma_{\mathcal{R}}(f)=0$.

3.1. Pseudo-Anosov maps. We start with a remark on the translation distance of a pseudo-Anosov map $f \in \operatorname{Mod}(S)$, for the action of $f$ on Teichmüller space equipped with the Thurston metric, which was investigated in [10]. By definition, there is a pair of transverse measured laminations $\left(\mu^{s}, \mu^{u}\right)$ binding the surface $S$ (which are called the stable and unstable measured laminations associated to $f$ ) satisfying

$$
f\left(\mu^{s}\right)=K \mu^{s}, f\left(\mu^{u}\right)=\frac{1}{K} \mu^{u},
$$

where $K=\lambda(f)$. By a result of Thurston (see [7] and [15]), both $\mu^{s}$ and $\mu^{u}$ are minimal and uniquely ergodic. Taking a sequence of simple closed curves to approximate $\mu^{u}$, it follows directly from the definition of the Thurston metric that

$$
\sigma(f) \geq \log \lambda(f) .
$$

Let us now endow $S$ with some hyperbolic structure. The complement of $S \backslash \mu^{u}$ consists of a finite number of ideal polygons. We adjoin a finite number of leaves to $\mu^{u}$ such that it becomes a complete geodesic lamination, which we denote by $\overline{\mu^{u}}$. (Note that $\overline{\mu^{u}}$ is not necessarily unique, but there are finitely many choices).

Using Thurston's shearing coordinates [16], there exists a unique hyperbolic structure $X \in \mathcal{T}(S)$ corresponding the the pair $\left(\overline{\mu^{u}}, \mu^{s}\right)$ such that $\mu^{s}$ is, up to equivalence, the horocyclic foliation of $\overline{\mu^{u}}$. Then, $\left\{\left(\overline{\mu^{u}}, e^{t} \mu^{s}\right)\right\}_{t \in \mathbf{R}}$ defines a stretch line on $\mathcal{T}(S)$, which is a geodesic ray of the Thurston metric passing through the point $X \cong\left(\overline{\mu^{u}}, \mu^{s}\right)$.

Since the map $f$ acts isometrically between the hyperbolic surfaces $X$ and $f(X)$, the image of the stretch line $\left\{\left(\overline{\mu^{u}}, e^{t} \mu^{s}\right)\right\}_{t \in \mathbf{R}}$ is the stretch line $\left\{\left(f\left(\overline{\mu^{u}}\right), e^{t} f\left(\mu^{s}\right)\right)\right\}_{t \in \mathbf{R}}$. Note that $f\left(\mu^{s}\right)=K \mu^{s}$. There exists an integer $n$ such that $f^{n}\left(\overline{\mu^{u}}\right)=\overline{\mu^{u}}$. It turns out that the stretch line $\left\{\left(\overline{\mu^{u}}, e^{t} \mu^{s}\right)\right\}_{t \in \mathbf{R}}$ is preserved by $f^{n}$ and the point $X \cong\left(\overline{\mu^{u}}, \mu^{s}\right)$ is transformed into $f^{n}(X) \cong\left(\lambda, K^{n} \mu^{s}\right)$. It follows from an argument of Bers [4] that the translation distance

$$
\sigma\left(f^{n}\right)=\log \lambda\left(f^{n}\right)=n \log \lambda(f) .
$$

If $f$ is reducible, by Corollary 3.3, the translation distance of $f$ is at most $\max _{\mathcal{R}} \sigma_{\mathcal{R}}(f)$, where $\mathcal{R}$ is taken over all reducible components of $f$. If $\mathcal{R}=R_{0} \cup$ $f\left(R_{0}\right) \cup \cdots \cup f^{k-1}\left(R_{0}\right)$ is a pseudo-Anosov component, then we can take a hyperbolic structure $X_{0} \in \mathcal{T}\left(R_{0}\right)$ such that $X_{0}$ lies on a stretch line preserved by some power 
$f^{n}$. We equip each $f^{j}\left(R_{0}\right)$ with the hyperbolic structure $f^{j}\left(X_{0}\right), 1 \leq j \leq k-1$. This shows that

$$
\sigma_{\mathcal{R}}\left(f^{n}\right)=n \log \lambda\left(\left.f\right|_{\mathcal{R}}\right)
$$

where $\left.f\right|_{\mathcal{R}}$ denotes the restriction of $f$ on $\mathcal{R}$.

Proof of Theorem 1.1. It follows directly from the definition of the Thurston metric that

$$
\sigma\left(f^{n}\right) \geq n \log \lambda(f)
$$

Since $f$ has finitely many reducible components, the theorem follows from the above discussion, by taking $n$ sufficiently large.

Remark 3.4. When $f$ has no pseudo-Anosov component, we have $\sigma(f)=0$. Whether the dilatation $\lambda(f)$ is equal to $\sigma(f)$, when $f$ is a general pseudo-Anosov map, remains an open question. Note that by Wolpert's inequality, $d_{\text {Th }} \leq 2 d_{\text {Teich }}$. As a result, we have

$$
\log \lambda(f) \leq \sigma(f) \leq 2 \log \lambda(f) .
$$

3.3. Proof of Lemma 3.2. In this subsection, we prove Lemma 3.2, which is used in $\S 3.1$. We also take $X_{L}$ to be a family of hyperbolic structures on $S$ such that the lengths of the curves in the curve system $\mathcal{C}$ are given by the vector $L$. As $L \rightarrow 0, X_{L} \rightarrow X_{0}$. As before, we denote the geodesic representative of $\mathcal{C}$ on $X_{L}$ by $\widehat{\mathcal{C}}$. By cutting $X_{L}$ along $\widehat{\mathcal{C}}$, we obtain finitely many subsurfaces with geodesic boundary, denoted by $\left\{X_{L}^{j}\right\}$. The mapping class $f$ acts on $X_{L}$, preserving the set $\widehat{\mathcal{C}}$ (with some power of Dehn twists around each component of $\widehat{\mathcal{C}}$ ).

Lemma 3.5. There is a uniform constant $K$ such that $f$ is homotopic to a $K$-quasiconformal mapping from $X_{L}$ to $f\left(X_{L}\right)$.

Proof. We use an argument of Bishop [5] to construct an explicit map between $X_{L}$ and $f\left(X_{L}\right)$ with the required properties. We choose a geodesic pants decomposition for each subsurface $X_{L}^{j}$. For each pair of pants with boundary curves $\left\{\alpha_{1}, \alpha_{2}, \alpha_{3}\right\}$ (the pair of pants is degenerate, some of the curves may be punctures), we can deform it into a new one such that the boundary lengths $\left(\ell_{\alpha_{1}}\left(X_{L}^{j}\right), \ell_{\alpha_{2}}\left(X_{L}^{j}\right), \ell_{\alpha_{3}}\left(X_{L}^{j}\right)\right)$ are replaced by $\left(\ell_{\alpha_{1}}\left(f\left(X_{L}^{j}\right)\right), \ell_{\alpha_{2}}\left(f\left(X_{L}^{j}\right)\right), \ell_{\alpha_{3}}\left(f\left(X_{L}^{j}\right)\right)\right)$. Bishop [5] proved that such a deformation can be constructed in such a way that the quasiconformal dilatation only depends on the upper bound of

$$
\max \left\{\left|\log \frac{\ell_{\alpha_{1}}\left(f\left(X_{L}^{j}\right)\right)}{\ell_{\alpha_{1}}\left(X_{L}^{j}\right)}\right|,\left|\log \frac{\ell_{\alpha_{2}}\left(f\left(X_{L}^{j}\right)\right)}{\ell_{\alpha_{2}}\left(X_{L}^{j}\right)}\right|,\left|\log \frac{\ell_{\alpha_{3}}\left(f\left(X_{L}^{j}\right)\right)}{\ell_{\alpha_{3}}\left(X_{L}^{j}\right)}\right|\right\} .
$$

Furthermore, we can glue all the new pairs of pants together (in the same topological pattern as before) with appropriate twists such that the resulting structure is $f\left(X_{L}^{j}\right)$. The gluing map is again a quasiconformal mapping, with dilatation controlled by (an upper bound of) the lengths and twists of the curves in the pants decomposition (see $[3])$.

We construct the above deformation for each $X_{L}^{j}$, and we obtain $f\left(X_{L}\right)$ by gluing the resulting surfaces $f\left(X_{L}^{1}\right), \cdots, f\left(X_{L}^{s}\right)$, in addition with some fixed power of Dehn twists around each component of $\widehat{\mathcal{C}}$. Note that as $L \rightarrow 0$, the hyperbolic structures $X_{L}$ are chosen such that on each component of $X_{L} \backslash \widehat{\mathcal{C}}$, the lengths and twists of some pants decomposition are fixed (or almost fixed). Therefore, the quasiconformal 
dilatation of the above deformations have a uniform upper bound which does not depend on the choice of $L$.

Consider any simple closed geodesic $\gamma$ on $X_{L}$ which intersects $\widehat{\mathcal{C}}$. Let $\gamma_{j}$ be a segment of $\gamma$ contained in a component, say $X_{L}^{j}$, of $X_{L} \backslash \widehat{\mathcal{C}}$. We assume that the two endpoints of $\gamma_{j}$ lie on $\beta_{1}$ and $\beta_{2}$, respectively. Denote the length of $\gamma_{j}$ by $L_{j}$.

It is convenient to work in the universal cover $\mathbf{H}^{2}$. As shown in Figure 2, the geodesic $\beta_{1}$ is lifted to the $y$-axis $\tilde{\beta}_{1}$, with endpoints 0 and $\infty$. We can choose a universal cover such that the point 1 is an endpoint of $\tilde{\beta}_{2}$, a lift of $\beta_{2}$, and the geodesic segment $\gamma_{j}$ is realized as a geodesic segment connecting $\tilde{\beta}_{1}$ and $\tilde{\beta}_{2}$. The geodesic representative of $\gamma_{j}$ on the subsurface $X_{j}^{L}$ (that is, the shortest geodesic segment on $X_{j}^{L}$ homotopic to $\gamma_{j}$, by allowing the endpoints of $\gamma_{j}$ to vary on the boundary of $X_{j}^{L}$ ) corresponds to the geodesic segment $\hat{\gamma}_{j}$, which intersects $\tilde{\beta}_{1}$ and $\tilde{\beta}_{2}$ perpendicularly. A lift of $\gamma$, denoted by $\tilde{\gamma}$, is also drawn in the figure, with endpoints $x_{1}$ and $x_{2}$.

One can see as in Figure 2 that the geodesic segments $\gamma_{j}, \hat{\gamma}_{j}$ together with $\tilde{\beta}_{1}$ and $\tilde{\beta}_{2}$ bound a geodesic quadrilateral.

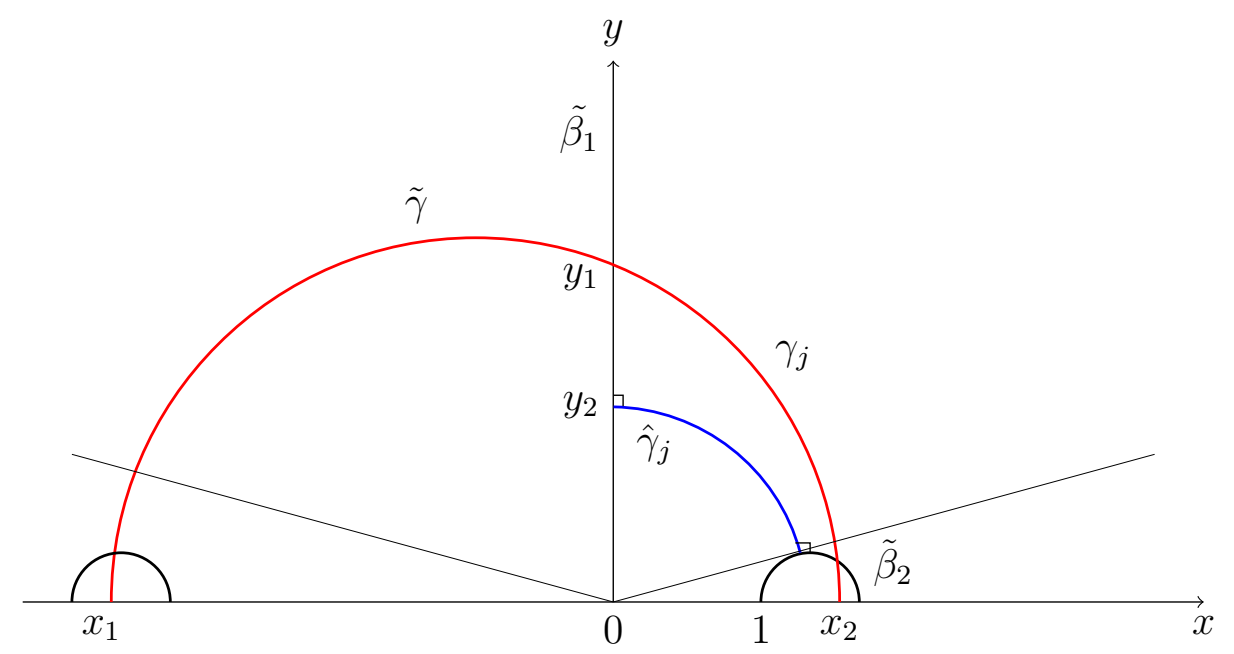

Figure 2.

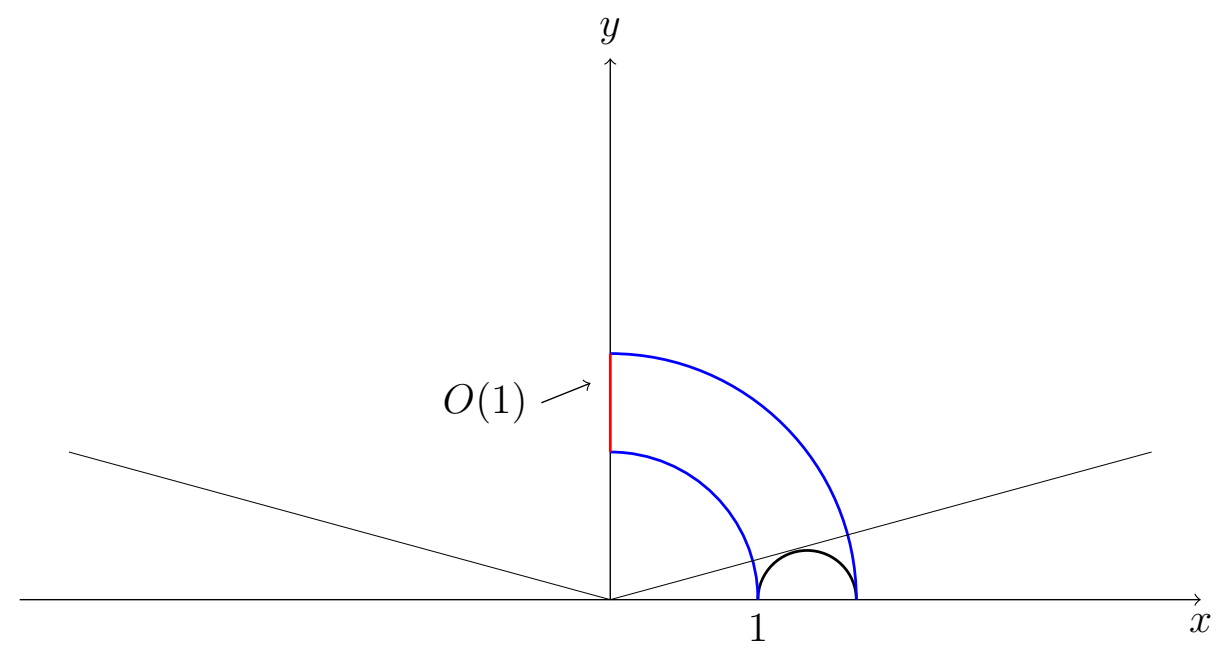

Figure 3 . 
The length of the sides on the left and right of the quadrilateral will be denoted by $a$ and $b$, respectively. Note that $a=\left|\log y_{2}-\log y_{1}\right|$. We apply the hyperbolic quadrilateral formula. (We refer to [11, Chapter 8] for the hyperbolic quadrilateral formula, and more generally for the hyperbolic geometry formulae used in the present paper):

$$
\cosh \left(L_{j}\right)=-\sinh (a) \sinh (b)+\cosh (a) \cosh (b) \cosh \left(\ell_{j}\right) .
$$

As $L \rightarrow 0$, both of $L_{j}$ and $\ell_{j}$ are sufficiently large. Thus we have the following approximation

$$
L_{j}=a+b+\ell_{j}+O(1) .
$$

We have to compare the above data with those of $f\left(X_{L}\right)$. We denote the corresponding quantities of $f\left(X_{L}\right)$ by $y_{1}^{\prime}, y_{2}^{\prime}, \cdots$. By Lemma 3.5, there is a $K$ quasiconformal mapping between $X_{L}$ and $f\left(X_{L}\right)$. Such a mapping can be lifted to a quasiconformal mapping $\tilde{f}$ on $\mathbf{H}^{2}$, such that it preserves the three points $0,1, \infty$. There is a uniform constant $M$ such that for any $p, q, r, s$ on $\partial \mathbf{H}^{2}$,

$$
\frac{1}{M}|(p, q, r, s)| \leq|(f(p), f(q), f(r), f(s))| \leq M|(p, q, r, s)| .
$$

This implies that

$$
\max \left\{1, \frac{x_{1}}{M}\right\} \leq f\left(x_{1}\right) \leq M x_{1}, \max \left\{\frac{\left|x_{2}\right|}{M}, 0\right\} \leq\left|f\left(x_{2}\right)\right| \leq M\left|x_{2}\right| .
$$

An estimate using Figure 3 easily shows that $\left|y_{2}-y_{2}^{\prime}\right|=O(1)$. In fact, since the collar neighborhood of $\beta_{1}$ is sufficiently large, the other endpoint of $\tilde{\beta}_{2}$ is close to 1 (this is also true for $f\left(\tilde{\beta}_{2}\right)$ ). Thus their projections on $\tilde{\beta}_{1}$ is near $i$, up to an uniformly bounded distance.

On the other hand, we have $\left|y_{1}-y_{1}^{\prime}\right|=O(1)$. This can be seen by a direct calculation. Note that the two endpoints of $\tilde{\gamma}$ are $x_{1}$ and $x_{2}$. It is easy to see that $y_{1}=\sqrt{\left|x_{1} x_{2}\right|}$. Under deformation by $\tilde{f}$, we have $y_{1}^{\prime}=\sqrt{\left|x_{1}^{\prime} x_{2}^{\prime}\right|}$.

As we noticed before,

$$
\frac{1}{M}\left|x_{1}\right| \leq\left|x_{1}^{\prime}\right| \leq M\left|x_{1}\right|, \quad \frac{1}{M}\left|x_{2}\right| \leq\left|x_{2}^{\prime}\right| \leq M\left|x_{2}\right| .
$$

This means that $\left|\log \frac{y_{1}^{\prime}}{y_{1}}\right| \leq \log M$. As a corollary, under the deformation $\tilde{f}, a$ is changed by a bounded amount, that is, $\left|a^{\prime}-a\right|=O(1)$. The same argument applies to $\left|b^{\prime}-b\right|$.

Applying the quadrilateral formula again

$$
\cosh \left(L_{j}^{\prime}\right)=-\sinh \left(a_{1}^{\prime}\right) \sinh \left(a_{2}^{\prime}\right)+\cosh \left(a_{1}^{\prime}\right) \cosh \left(a_{2}^{\prime}\right) \cosh \left(\ell_{j}^{\prime}\right) .
$$

The above computations show that

$$
\begin{aligned}
L_{j} & =a+b+\ell_{j}+O(1), \\
L_{j}^{\prime} & =a^{\prime}+b^{\prime}++\ell_{j}^{\prime}+O(1), \\
\left|a-a^{\prime}\right| & =O(1), \quad\left|b-b^{\prime}\right|=O(1) .
\end{aligned}
$$

Thus (using the fact that $L_{j}, \ell_{j} \rightarrow \infty$ as $L \rightarrow 0$ )

$$
\frac{L_{j}^{\prime}}{L_{j}} \leq(1+o(1)) \max \left\{1, \frac{\ell_{j}^{\prime}}{\ell_{j}}\right\}
$$

This completes the proof of Lemma 3.2. 


\section{References}

[1] Abikoff, W.: The real analytic theory of Teichmüller space. - Lecture Notes in Math. 820, Springer-Verlag, 1980.

[2] Alessandrini, D., L. Liu, A. Papadopoulos, and W. Su: The horofunction compactification of the arc metric on Teichmüller space. - arXiv:1411.6208.

[3] Alessandrini, D., L. Liu, A. Papadopoulos, W. Su, and Z. Sun: On Fenchel-Nielsen coordinates on Teichmüller spaces of surfaces of infinite type. - Ann. Acad. Sci. Fenn. Math. 36, 2011, 621-659.

[4] Bers, L.: An extremal problem for quasiconformal mappings and a theorem by Thurston. Acta Math. 141, 1978, 73-98.

[5] Bishop, C. J.: Quasiconformal mappings of $Y$-pieces. - Rev. Mat. Iberoam. 18:3, 2002, 627652.

[6] Buser, P.: Geometry and spectra of compact Riemann surfaces. - Progr. Math. 106, Birkhäuser Boston, Inc., Boston, MA, 1992.

[7] Fathi, A., F. Laudenbach, and V. Poénaru: Travaux de Thurston sur les surfaces. Astérisque 66-67, 1979; English transl.: Thurston's work on surfaces. - Princeton Univ. Press, 2012.

[8] Liu, L., A. Papadopoulos, W. Su, and G. ThÉret: On length spectrum metrics and weak metrics on Teichmüller spaces of surfaces with boundary. - Ann. Acad. Sci. Fenn. Math. 35:1, 255-274, 2010.

[9] Liu, L., A. Papadopoulos, W. Su, and G. ThéRet: Length spectra and the Teichmüller metric for surfaces with boundary. - Monats. Math. 161:3, 2010, 295-311.

[10] Liu, L., A. Papadopoulos, W. Su, and G. ThÉret: On the classification of mapping class actions on Thurston's asymmetric metric. - Math. Proc. Cambridge Philos. Soc. 155:03, 2013, 499-515.

[11] Marden, A.: Outer circles: An introduction to hyperbolic 3-manifolds. - Cambridge Univ. Press, Cambridge, 2007.

[12] Mondello, G.: A criterion of convergence in the augmented Teichmüller space. - Bull. Lond. Math. Soc. 41, 2009, 733-746.

[13] Papadopoulos, A., and G. Théret: Some Lipschitz maps between hyperbolic surfaces with applications to Teichmüller theory. - Geom. Dedicata 150:1, 2011, 233-247.

[14] Papadopoulos, A., and G. ThÉret: Shortening all the simple closed geodesics on surfaces with boundary. - Proc. Amer. Math. Soc. 138, 2010, 1775-1784.

[15] Thurston, W. P.: On the geometry and dynamics of diffeomorphisms of surfaces. - Bull. Amer. Math. Soc. 19, 1988, 417-431.

[16] Thurston, W.P.: Minimal stretch maps between hyperbolic surfaces. - Preprint, 1986, arxiv:math/9801039v1.

[17] Walsh, C.: The horoboundary and isometry groups of Thurston's Lipschitz metric. - In: Handbook of Teichmüller theory IV, ed. A. Papadopoulos, Eur. Math. Soc., 2014, 327-353.

[18] Wolpert, S.: Behavior of geodesic-length functions on Teichmüller space. - J. Differential Geom. 79:2, 2008, 277-334.

[19] Wolpert, S.: Geometry of the Weil-Petersson completion of Teichmüller space. - In: Surveys in differential geometry, ed. S.-T. Yau, Lectures on geometry and topology held in honor of Calabi, Lawson, Siu, and Uhlenbeck at Harvard University, Cambridge, MA, USA, May 3-5, 2002, International Press, Somerville, MA, 2003, 357-393. 